\title{
Carbon dioxide Angiography-Guided Renal-Related Interventions in Patients with Takayasu Arteritis and Renal Insufficiency
}

\author{
Sujith Chacko $^{1} \cdot$ George Joseph $^{1}$ (D) Viji Thomson ${ }^{1} \cdot$ Paul George $^{1} \cdot$ \\ Oommen George ${ }^{1} \cdot$ Debashish Danda $^{2}$
}

Received: 26 October 2017/Accepted: 8 March 2018/Published online: 16 March 2018

(C) The Author(s) 2018

\begin{abstract}
Background Use of iodinated contrast agents for angiography in patients with renal insufficiency risks further deterioration of renal function and its adverse sequelae.

Objective To study the effectiveness and safety of carbon dioxide $\left(\mathrm{CO}_{2}\right)$ angiography in guiding percutaneous renalrelated interventions in patients with Takayasu arteritis and renal insufficiency.

Methods Data on $\mathrm{CO}_{2}$ angiography-guided interventions were obtained from a 23-year database of 692 Takayasu arteritis patients who underwent percutaneous interventions and were analyzed retrospectively. Follow-up data were also obtained. The $\mathrm{CO}_{2}$ angiography system used was developed in-house and was pressure-driven.

Results Seven patients (6 female, age 16-59 years, baseline serum creatinine $1.62-4.55 \mathrm{mg} / \mathrm{dl}$, estimated glomerular filtration rate $12.2-36.9 \mathrm{ml} / \mathrm{min} / 1.73 \mathrm{~m}^{2}$ ) underwent $\mathrm{CO}_{2}$ angiography-guided interventions: five underwent angioplasty or stenting to treat six stenotic/occluded renal arteries, one underwent extensive endovascular repair for spontaneous focal abdominal aortic dissection with false lumen aneurysm and aorto-iliac true lumen narrowing, and one underwent balloon dilatation of previously deployed aortic stents used to treat aortic occlusion at two levels. Follow-up (median 5 years, range 2 months-16 years) was obtained in all patients. All the procedures were successful and resulted in relief of
\end{abstract}

George Joseph

joseph59@gmail.com

1 Department of Cardiology, Christian Medical College, Vellore 632004, India

2 Department of Rheumatology, Christian Medical College, Vellore, India symptoms, better blood pressure control, improvement in left ventricular systolic function and recovery or stabilization of renal function. There were no early or late complications related to $\mathrm{CO}_{2}$ angiography. Three renal lesions that had restenosis at follow-up were managed successfully by repeat intervention.

Conclusion $\mathrm{CO}_{2}$ angiography-guided renal-related interventions are effective and safe in patients with Takayasu arteritis and renal insufficiency; they significantly improve the care of such patients.

Keywords Renal insufficiency $\cdot$ Renal failure ·

Takayasu arteritis - Carbon dioxide - Angioplasty ·

Stent · Renal artery stenosis - Aortic stenosis .

Dissection · Pseudoaneurysm

\section{Introduction}

Carbon dioxide $\left(\mathrm{CO}_{2}\right)$ has been used as an intra-arterial contrast agent during angiography for more than four decades [1]. Renal insufficiency is an important indication for the use of $\mathrm{CO}_{2}$ angiography, given the absence of nephrotoxicity with this agent [2]. Takayasu arteritis (TA), a chronic idiopathic granulomatous large vessel vasculitis affecting the aorta and its main branches [3], can produce renal insufficiency [4], mainly by causing renal artery stenosis [5]. At our center, prevalence of renal dysfunction in 118 patients with TA presenting during the period 1963-1977 was $13.6 \%$ [6]. A recent study by Li et al. [4] showed an overall prevalence of renal dysfunction of $11.4 \%$ in 411 patients with TA. There are no reports of the 
use of $\mathrm{CO}_{2}$ angiography-guided interventions in TA apart from a single case report from our center in 2003 [7]; the present study describes our 16-year single-center experience with $\mathrm{CO}_{2}$ angiography in guiding renal-related percutaneous interventions in patients with TA.

\section{Methods}

\section{Patients}

Data on percutaneous interventions performed on patients with TA at our center (a large tertiary care referral hospital in South India) that were collected prospectively over 23 years and archived digitally were scrutinized. Cases where $\mathrm{CO}_{2}$ angiography was utilized to guide percutaneous interventions were selected, and the relevant case records and angiographic images were analyzed. Follow-up was obtained in all patients at 6- to 12-month intervals if stable, but more frequently when indicated. Clinical status, imaging information and additional procedures done at follow-up were studied. All procedures were performed after obtaining written informed consent. The institutional review board approved this retrospective study. For this type of study formal consent is not required.

\section{Carbon dioxide Angiography System}

The $\mathrm{CO}_{2}$ angiography system used (Fig. 1A) was developed in-house and has been operational for the last 17 years. It has no moving parts, and $\mathrm{CO}_{2}$ injection is pressure-driven. Pressurized medical grade $\mathrm{CO}_{2}$ obtained from a storage cylinder is passed through a Millipore filter into a disposable $50 \mathrm{ml}$ plastic collection syringe that is held firmly between fixed restraints on a metal platform. The piston of the collection syringe is variably restrained by an assembly of swivelling blocks; the volume of the syringe is determined by the number of blocks in use in the piston stopper assembly. A two-way stopcock attached to the collection syringe nozzle ensures that at no stage can the flow of $\mathrm{CO}_{2}$ bypass the syringe and go directly from cylinder to patient. Gas pressure in the collection syringe is kept at $4 \mathrm{~kg} / \mathrm{cm}^{2}$ (nearly $4 \mathrm{~atm}$ ) by appropriately adjusting the knob in the dual pressure gauge assembly which separately indicates cylinder (inflow) and tubing (outflow) pressures. At this setting, the volume of $\mathrm{CO}_{2}$ delivered into the vessel is approximately three times the selected volume in the collection syringe; this can be verified by trial injection of $\mathrm{CO}_{2}$ into a collapsed plastic bag attached to the three-way stopcock along the tubing. Prior to angiography, the tubing and angiographic catheter are flushed with $\mathrm{CO}_{2}$ to remove air and avoid explosive delivery of $\mathrm{CO}_{2}$. For $\mathrm{CO}_{2}$ injection, the two-way stopcock is turned $90^{\circ}$ to allow
$\mathrm{CO}_{2}$ to flow from the collection syringe to the patient. Standard protocols were used for patient preparation and digital subtraction angiography. Typically, the collection syringe volume was set at $20 \mathrm{ml}$ for flush aortograms (to deliver $60 \mathrm{ml}$ of $\mathrm{CO}_{2}$ ) and $10 \mathrm{ml}$ for selective angiograms (to deliver $30 \mathrm{ml}$ of $\mathrm{CO}_{2}$ ). The quality of images obtained using this $\mathrm{CO}_{2}$ angiography system compared favorably with that obtained with $50 \%$ iodixanol, a diluted alternative contrast agent, that is sometimes used in renal insufficiency (Fig. 1B, C).

\section{Results}

Of 692 patients with TA who underwent percutaneous interventions to treat 1834 diseased arteries (including 425 renal arteries) over a 23-year period, seven patients (1.01\%) underwent $\mathrm{CO}_{2}$ angiography-guided percutaneous interventions because of renal insufficiency (Table 1). All seven patients ( 1 male/ 6 female, mean age 38 years) met both the American College of Rheumatology [8] and the modified Ishikawa (clinical) criteria [9] for the diagnosis of TA; all were hypertensive on multiple medications. All patients were symptomatic at presentation, often with recent worsening.

\section{Renal Interventions}

Five patients with significant renal artery stenosis underwent $\mathrm{CO}_{2}$ angiography-guided renal angioplasty or stenting using percutaneous femoral arterial access (Table 1, cases 1-5; Fig. 2). In cases 1, 2 and 4, the renal artery was engaged with a $7 \mathrm{~F}$ renal-guiding catheter and the stenotic lesion was crossed with a 0.018 -inch nitinol guidewire. In cases 3 and 5 , the renal arteries were engaged with a $6 \mathrm{~F}$ Judkins Right diagnostic catheter and a 0.035-inch angled hydrophilic guidewire was used to cross the renal artery lesion; this was replaced with a 0.035 -inch stiff guidewire with 1-cm soft tip over which a long 7F femoral sheath was advanced up to the renal artery ostium. Balloon-expandable bare metal stents were deployed in all the lesions except in case 5 where a stent had been deployed earlier. Balloondilatation pressures of 8 to 14 atm were used. A satisfactory result was obtained in all vessels without complications.

\section{Aortic Interventions}

Two patients underwent $\mathrm{CO}_{2}$ angiography-guided aortic and ancillary interventions with successful outcomes and without complications (Table 1, cases 6, 7; Fig. 3).

In case 6 , extensive endovascular repair was performed for spontaneous focal abdominal aortic dissection with 


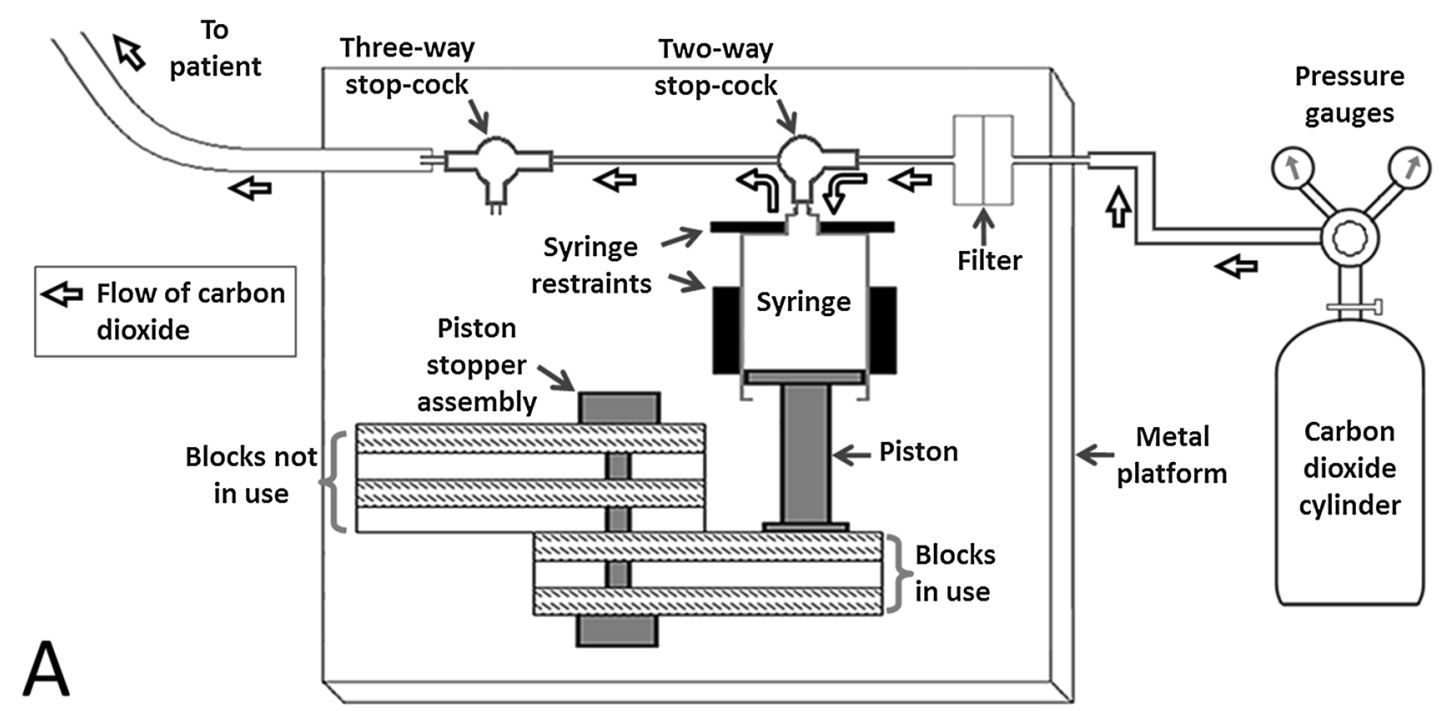

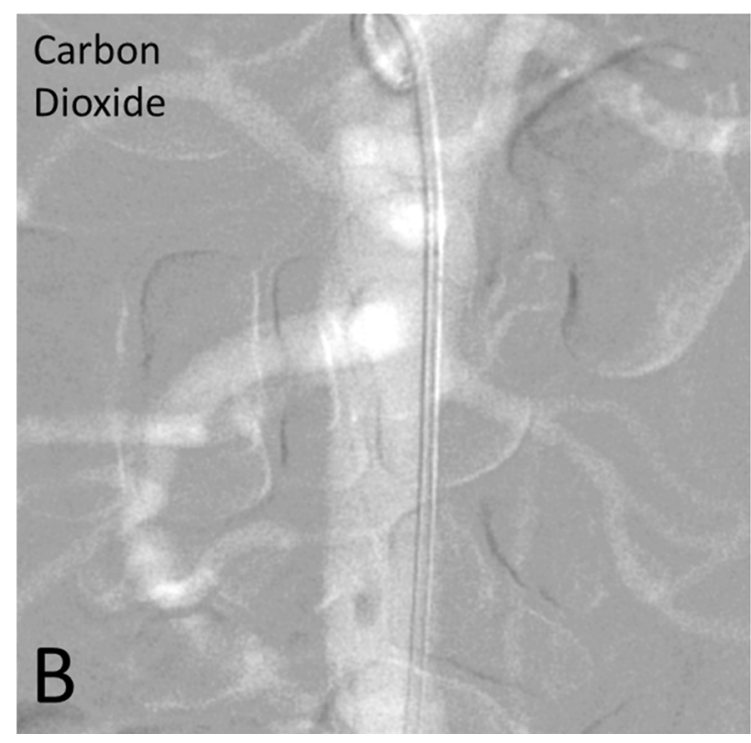

Fig. 1 A Diagrammatic representation of the apparatus used for carbon dioxide angiography. Every alternate block of the piston stopper assembly has been left un-shaded to reveal the perpendicular rod around which the blocks can swivel $180^{\circ}$; all the blocks are

false lumen aneurysm formation and aorto-iliac true lumen narrowing with $103 \mathrm{mmHg}$ systolic pressure gradient. The celiac and left renal arteries were the only patent visceral aortic branches. In the first sitting, aorto-iliac stenting was performed with a covered stent in the infra-renal aorta and bare metal stents in both common iliac arteries. In a second sitting 7 days later, a tapered aorto-uni-iliac endograft was deployed in the abdominal aorta starting above the upper limit of the aortic dissection (at the celiac artery ostium level) and overlapping the earlier deployed stent below. Flow into the left renal artery was preserved by constructing a chimney graft. Flow into the celiac artery was preserved using a bare metal stent deployed in chimney fashion. A satisfactory result was obtained with exclusion

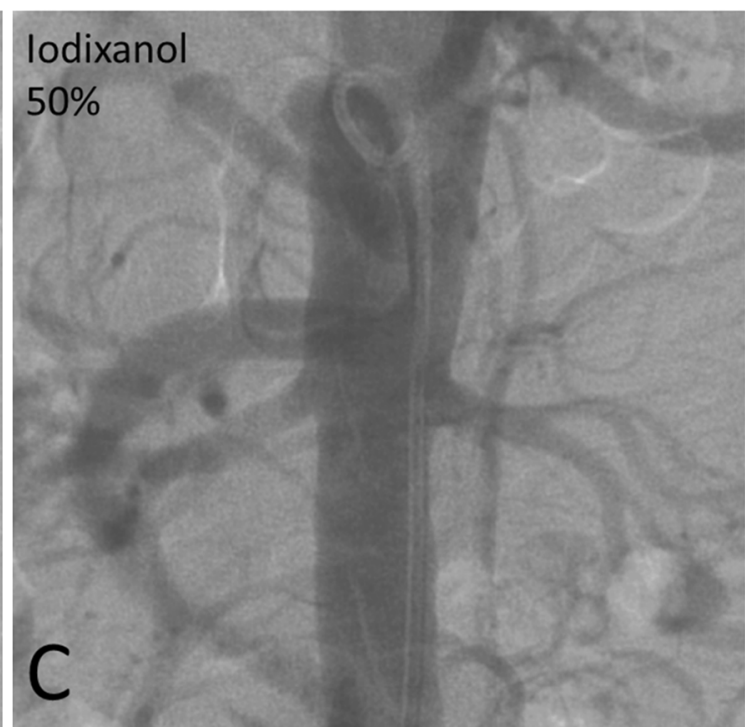

identical. B, C. Consecutive abdominal aortograms performed in antero-posterior projection in a patient with renal failure using carbon dioxide and $50 \%$ iodixanol, respectively, with identical settings of digital subtraction angiography

of the false lumen aneurysm, abolition of the aorto-iliac pressure gradient and preserved flow into the left renal and celiac arteries.

Case 7 had presented 7 months earlier with occlusions of both renal arteries and of the aorta at two levels; the aortic occlusions were recanalized and stented with balloon-expandable stents, but the lesions were resistant to dilatation, and only partial opening was achieved with a 9-mm-diameter non-compliant balloon. Renal function had subsequently deteriorated, and in the present sitting $\mathrm{CO}_{2}$ angiography-guided balloon dilatation of both stents was performed using a 10-mm-diameter non-compliant balloon which resulted in further stent expansion and reduction in the aortic pressure gradient. This provided a sufficient 


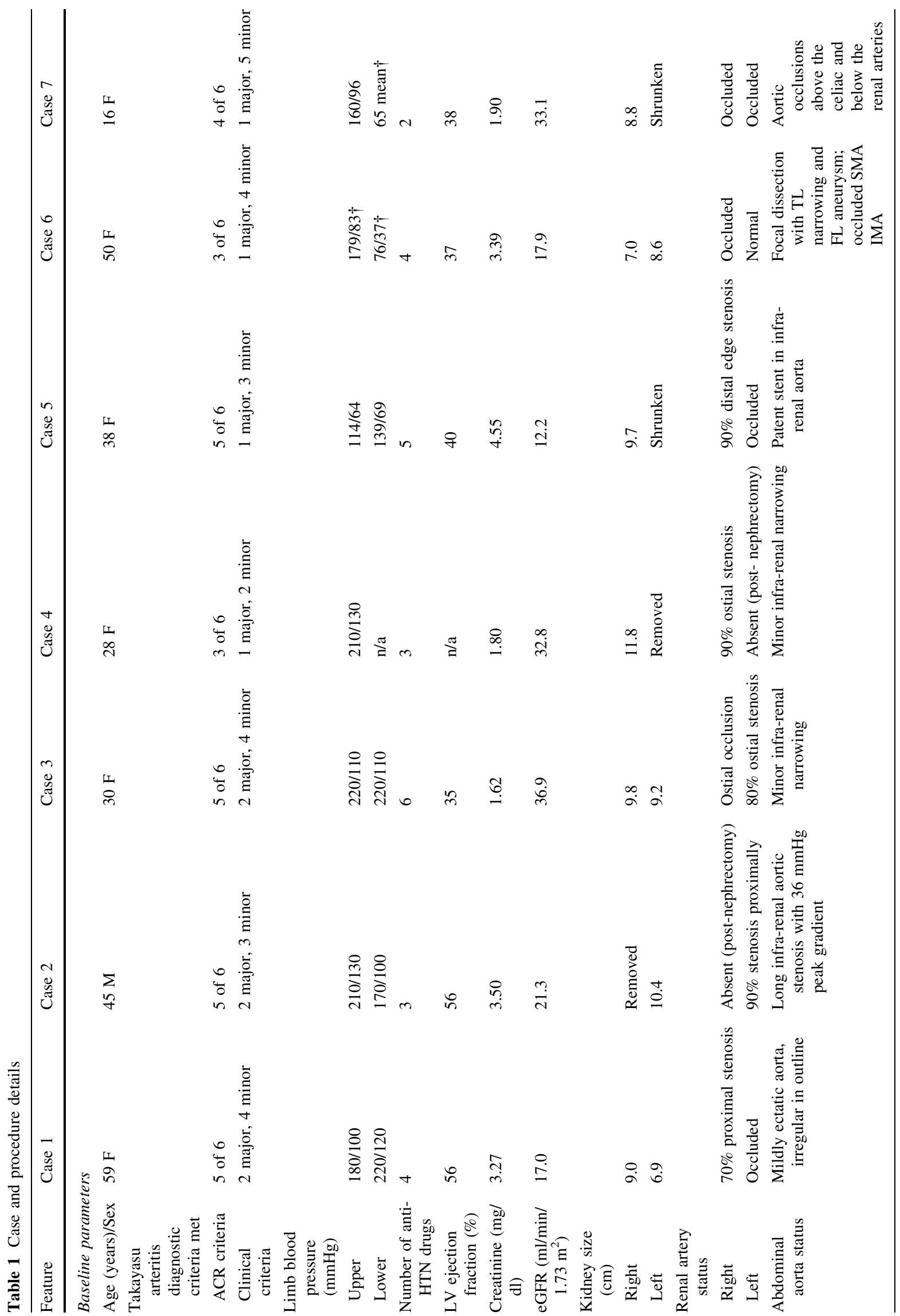




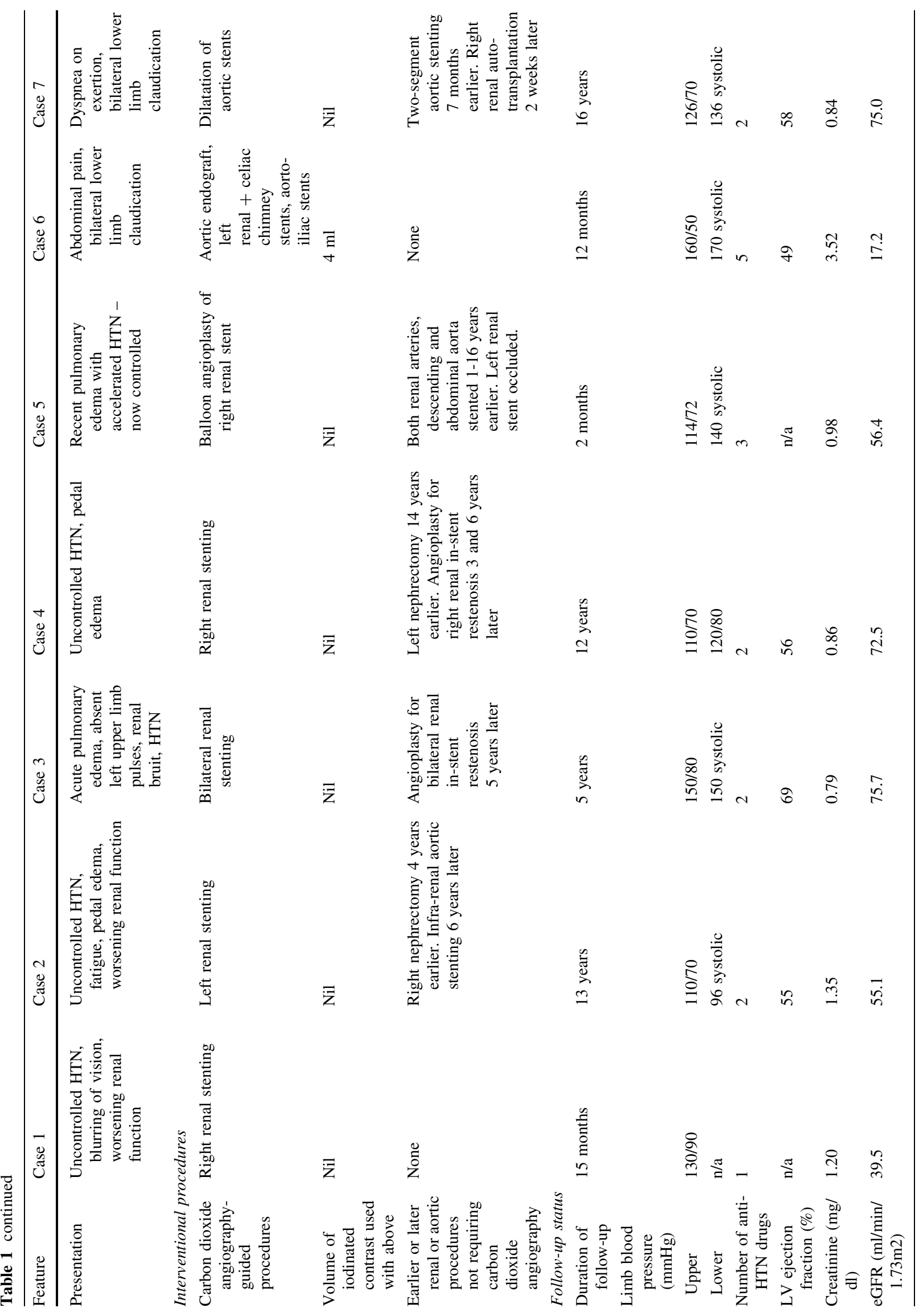


pressure head for the right kidney to be auto-transplanted 2 weeks later to the right iliac fossa with the right renal artery being anastomosed end-to-end to the right internal iliac artery.

\section{Outcome and Follow-Up}

$\mathrm{CO}_{2}$ angiography provided sufficiently clear visualization of the vascular anatomy and enabled achievement of successful outcomes in all the patients. Some patients felt mild transient abdominal discomfort immediately after $\mathrm{CO}_{2}$ injection, but none had nausea, vomiting, hypotension, narcosis or air contamination-related problems. There were no late complications related to $\mathrm{CO}_{2}$ angiography. All patients were followed-up after the $\mathrm{CO}_{2}$ angiography-guided intervention (median duration 5 years, range 2 months to 16 years; Table 1). None of the patients had deterioration in renal function or required dialysis; rather, renal function improved in 6 patients and stabilized in one. All patients experienced resolution of their symptoms. Blood pressure control improved, as also left ventricular systolic function that was initially depressed in some patients. Restenotic renal artery lesions seen at follow-up in cases 3 and 4 were treated by balloon dilatation; in case 3 this produced distal edge dissection in the right renal artery and required deployment of a stent. In case 7 , progressive dilatation of the aortic stents was performed during subsequent follow-up visits.

\section{Comparison with Iodinated Contrast}

During the same period of time, 46 TA patients with elevated serum creatinine levels $(>1.4 \mathrm{mg} / \mathrm{dl})$ underwent interventions using iodinated contrast after intravenous hydration; of these, none with serum creatinine levels below $3.0 \mathrm{mg} / \mathrm{dl}$ developed sustained worsening of renal function or required dialysis, but one of two patients with serum creatinine $\geq 3.0 \mathrm{mg} / \mathrm{dl}$ required long-term dialysis. On the other hand, none of the TA patients who underwent $\mathrm{CO}_{2}$ angiography-guided interventions, including four with serum creatinine $\geq 3.0 \mathrm{mg} / \mathrm{dl}$, had worsening of renal function or required dialysis.

\section{Discussion}

Contrast-induced nephropathy is one of the most common causes of hospital-acquired renal insufficiency and is associated with a mortality of $14 \%$; pre-existing renal insufficiency poses the greatest risk for developing this condition [10]. Absence of nephrotoxicity is perhaps the biggest advantage $\mathrm{CO}_{2}$ provides as a contrast agent, though it has several other benefits including being non-allergic 

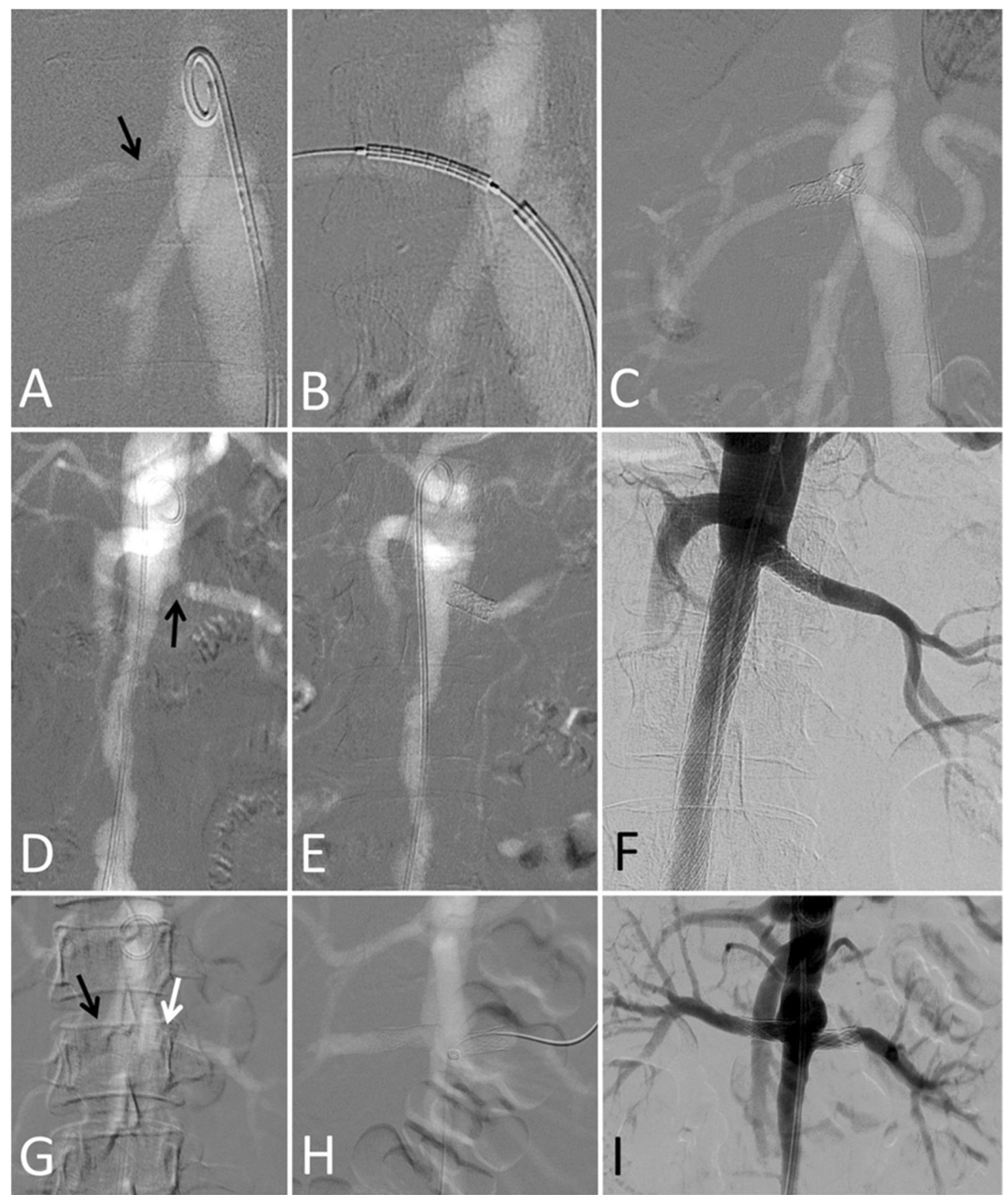

Fig. 2 Carbon dioxide-guided renal artery interventions in patients with Takayasu arteritis and renal insufficiency. All images are carbon dioxide angiograms unless stated otherwise. A to C. Case 1. Baseline angiogram (A) shows right renal artery stenosis (arrow). After stent positioning (B) and deployment, the final angiogram (C) showed a good outcome. D to F. Case 2. Baseline angiogram (D) shows ostial left renal artery stenosis (arrow) and long infra-renal aorta narrowing. Renal function normalized after left renal stenting (E). Conventional

and inexpensive, having low viscosity, allowing use of unlimited total volumes and not being diluted by blood [11]. With modern technology such as high-resolution angiogram $(\mathbf{F})$ obtained 11 years later (5 years after interval infrarenal aortic stenting) shows good long-term outcome. G to I. Case 3. Baseline angiogram $(\mathbf{G})$ shows right renal artery occlusion (black arrow) and left renal artery stenosis (white arrow). Bilateral renal artery stenting was performed $(\mathbf{H})$ leading to normalization of renal function. Conventional angiogram done 4 months later (I) shows good short-term outcome

digital subtraction angiography, stacking software, tilting tables and reliable delivery systems, the quality of images obtained with $\mathrm{CO}_{2}$ angiography has improved considerably 


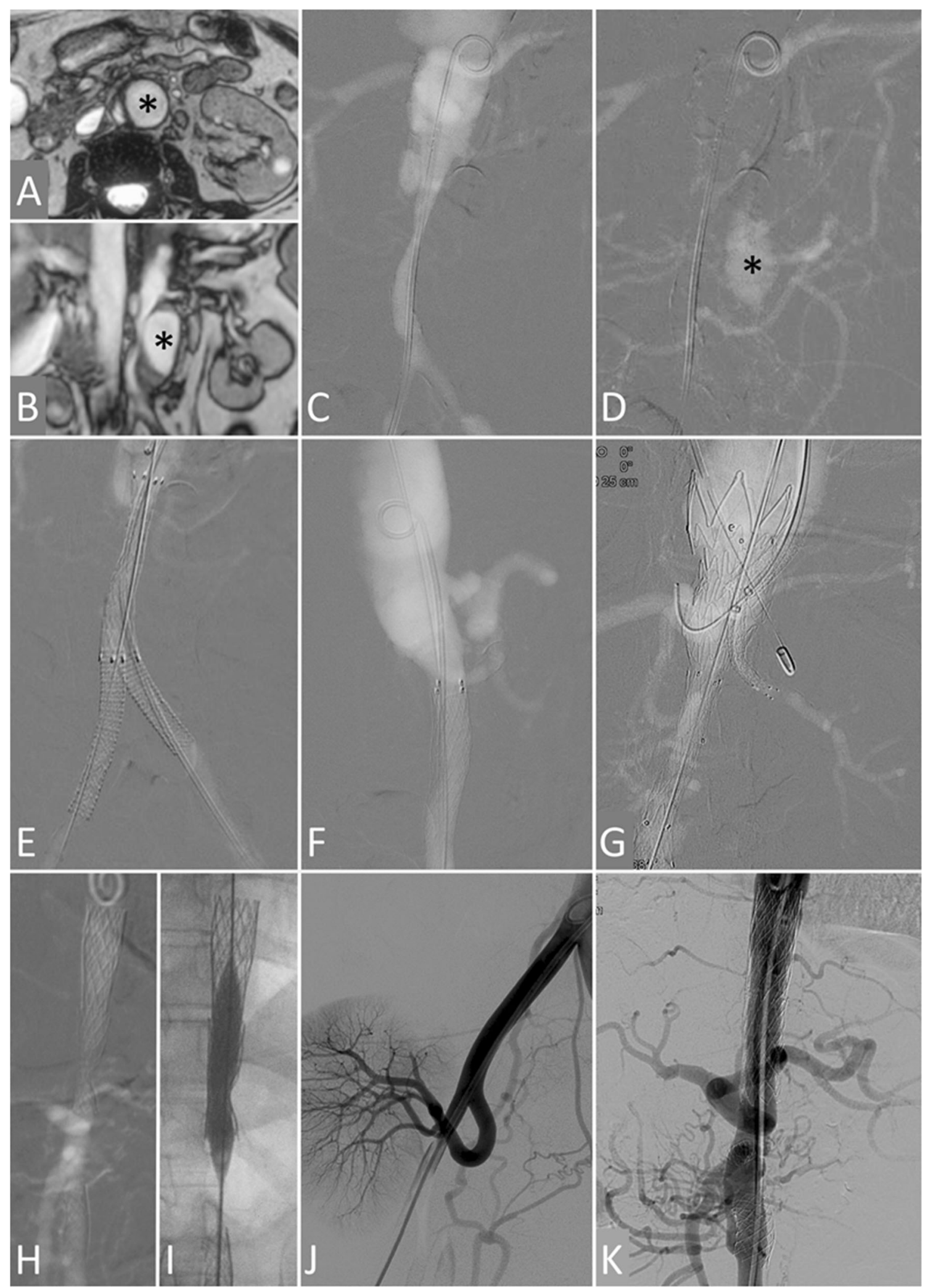


4Fig. 3 Carbon dioxide-guided aortic and ancillary interventions in patients with Takayasu arteritis and renal insufficiency. A to G. Case 6. Magnetic resonance angiograms in transverse (A) and coronal (B) planes and carbon dioxide angiogram early (C) and late (D) frames in antero-posterior (AP) projection show a single (left) renal artery, focal abdominal aortic dissection, narrowing of the infrarenal aorta and both common iliac arteries and a large false lumen aneurysm on the left lateral aspect of infra-renal aorta (black asterisk). Carbon dioxide AP (E) and lateral $(\mathbf{F})$ angiograms after infra-renal aortic and bilateral iliac artery stenting show relief of stenosis; the superior mesenteric and right renal arteries are not visualized. Carbon dioxide AP angiogram $(\mathbf{G})$ after deployment of a tapered endograft in the abdominal aorta and chimney grafts in the left renal and superior mesenteric arteries shows patency of these arteries; the false lumen aneurysm was no longer visualized in the late frames. H to K. Case 7. Carbon dioxide AP angiogram $(\mathbf{H})$ obtained 7 months after stenting of the lower thoracic-upper abdominal and infra-renal aorta (renal function had deteriorated since then) shows residual stenosis and nonvisualization of the renal arteries. The stents were further expanded by balloon dilatation (I), and the right kidney was auto-transplanted (J) resulting is normalization of renal function. Conventional AP aortogram (K) obtained 11 years later shows widely patent aortic stents

[2]. In many cases, such as the ones presented in this report, the entire percutaneous intervention can be performed without use of iodinated contrast. In case 6 , a single angiogram using $4 \mathrm{ml}$ of iodinated contrast diluted with saline was used to confirm adequate collateral flow from the celiac artery to the superior and inferior mesenteric artery territories; this was done before deploying aortic endografts across the ostia of these already occluded vessels because recanalization of these vessels would no longer be possible. An important prerequisite for successful $\mathrm{CO}_{2}$ angiography is gentle, controlled, non-explosive delivery of the gas using a closed and ideally non-pressurized system; this can be achieved by use a series of oneway valves with a flaccid reservoir or blood bag $[11,12]$. The $\mathrm{CO}_{2}$ angiography system used in this study was a pressurized system, but with adequate attention to outflow pressure and syringe volume and by purging the system with $\mathrm{CO}_{2}$ gas before use, no significant problems have been encountered; the system has worked well in more than 100 patients over 17 years, is simple, inexpensive and easy to use and has guided aorto-iliac, lower extremity, venous and complex endovascular procedures.

Renal insufficiency is not uncommon in TA despite these patients being young and having few co-morbidities $[4,6]$. Renal insufficiency in TA is usually attributed to renal ischemia caused by vascular obstruction and renal parenchymal damage induced by systemic hypertension; glomerular disease is considered exceptional [13]. Recent clinical studies $[5,14]$ support this contention. Hong et al. [5] found that $9.7 \%$ of TA patients with renal artery involvement developed chronic renal insufficiency over a 90-month follow-up period. Similarly, Obiagwu et al. [14] showed that renal artery revascularization procedures were effective in salvaging renal function in children with TAinduced renal artery stenosis. However, an autopsy study [15] on 25 TA patients showed that whereas non-specific, ischemic and/or hypertensive glomerular changes were present in $44 \%$ of kidney specimens, $56 \%$ showed specific glomerular pathologies, most commonly diffuse mesangial proliferative glomerulonephritis; extent of large arterial inflammatory infiltrates assessed by morphometric analysis was most in the latter condition suggesting a relationship between the two phenomena.

This report highlights some characteristic features of percutaneous interventions in TA. Firstly, the high rate of restenosis seen after bare metal renal artery stenting in TA. Secondly, repeat intervention, mostly balloon angioplasty alone, is usually successful in dealing with the problem of restenosis. Thirdly, stenotic lesions in TA may be very resistant to dilatation, but can be progressively opened up with serial balloon dilatations over multiple sittings. Lastly, spontaneous dissections and aneurysms seen in TA can be effectively treated by endovascular techniques with minimal morbidity.

\section{Conclusion}

Renal-related percutaneous interventions can be effectively and safely performed guided by $\mathrm{CO}_{2}$ angiography in patients with TA and renal insufficiency. Such procedures result in relief of symptoms, better blood pressure control, improvement in left ventricular systolic function and recovery or stabilization of renal function. $\mathrm{CO}_{2}$ angiography is a useful adjunct to the interventional armamentarium available to treat patients with TA and renal insufficiency.

\section{Compliance with Ethical Standards}

Conflict of interest All the authors declare that they have no conflict of interest.

Open Access This article is distributed under the terms of the Creative Commons Attribution 4.0 International License (http:// creativecommons.org/licenses/by/4.0/), which permits unrestricted use, distribution, and reproduction in any medium, provided you give appropriate credit to the original author(s) and the source, provide a link to the Creative Commons license, and indicate if changes were made.

\section{References}

1. Hawkins IF, Caridi JG. Carbon dioxide $\left(\mathrm{CO}_{2}\right)$ digital subtraction angiography: 26 year experience at the University of Florida. Eur Radiol. 1998;8:391-402. 
2. Hawkins IF, Cho KJ, Caridi JG. Carbon dioxide in angiography to reduce the risk of contrast-induced nephropathy. Radiol Clin North Am. 2009;47:813-25.

3. Kerr GS, Hallahan CW, Giordano J, Leavitt RY, Fauci AS, Rottem M, Hoffman GS. Takayasu arteritis. Ann Intern Med. 1994;120:919-29.

4. Li J, Li H, Sun F, Chen Z, Yang Y, Zhao J, Li M, Tian X, Zeng $\mathrm{X}$. Clinical characteristics of heart involvement in Chinese patients with Takayasu arteritis. J Rheumatol. 2017 Aug 15. https://doi.org/10.3899/jrheum.161514. Epub ahead of print.

5. Hong S, Ghang B, Kim YG, Lee CK, Yoo B. Long-term outcomes of renal artery involvement in Takayasu arteritis. J Rheumatol. 2017;44:466-72.

6. Alurkar VM, Cherian G, Sukumar IP, Krishnaswami S, Abraham KA, Raju AR. Non-specific aortoarteritis-a clinical profile. Christian Medical College Alumni Journal. 1978; XII: 5-14.

7. Chandy ST, John B, Kamath P, John GT. Exclusive carbon dioxide-guided renal artery stenting in a case of Takayasu's arteritis with a solitary functioning kidney. Indian Heart J. 2003;55:272-4.

8. Arend WP, et al. The American College of Rheumatology 1990 criteria for the classification of Takayasu arteritis. Arthritis Rheum. 1990;33:1129-34.
9. Sharma BK, Jain S, Suri S, Numano F. Diagnostic criteria for Takayasu arteritis. Int J Cardiol. 1996;54(Suppl):S141-7.

10. Nash K, Hafeez A, Hou S. Hospital-acquired renal insufficiency. Am J Kidney Dis. 2002;39:930-6.

11. Caridi JG, Cho KJ, Fauria C, Eghbalieh N. Carbon dioxide digital subtraction angiography (CO2 DSA): a comprehensive user guide for all operators. Vascu Dis Manag. 2014;11(10):E221-56.

12. Cherian MP, Mehta P, Gupta P, Kalyanpur TM, Jayesh SR, Rupa R. Technical note: a simple and effective $\mathrm{CO}_{2}$ delivery system for angiography using a blood bag. Indian $\mathrm{J}$ Radiol Imaging. 2009;19:203-5.

13. Boubaker K, Kaaroud H, Goucha R, Kheder A. Renal injury in Takayasu's arteritis. Nephrol Ther. 2014;10:451-6.

14. Obiagwu PN, Gajjar P, McCulloch M, Scott C, Numanoglu A, Nourse P. Salvageability of renal function following renal revascularisation in children with Takayasu's arteritis-induced renal artery stenosis. S Afr Med J. 2016;106:813-6.

15. de Pablo P, García-Torres R, Uribe N, Ramón G, Nava A, Silveira LH, Amezcua-Guerra LM, Martínez-Lavín M, Pineda C. Kidney involvement in Takayasu arteritis. Clin Exp Rheumatol. 2007;25(1 Suppl 44):S10-4. 